\title{
Defining outcome after meniscal allograft transplantation: Is buying time a valid measure of success?
}

\author{
Tim Spalding ${ }^{1} \cdot$ Alan Getgood $^{2}$
}

Published online: 22 April 2016

(C) European Society of Sports Traumatology, Knee Surgery, Arthroscopy (ESSKA) 2016

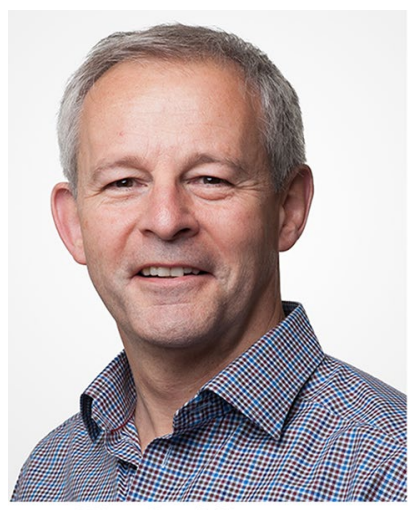

Tim Spalding

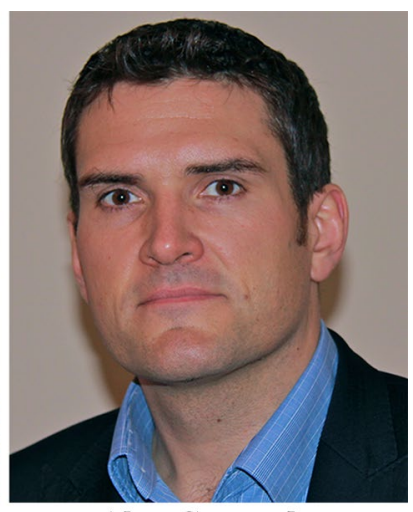

Alan Getgood
Loss of the meniscus leads to osteoarthritis of the knee with associated pain and deterioration in function and quality of life. As such, the renewed interest in meniscal preservation, as highlighted in this current issue, should be at the forefront of a knee surgeon's mind when treating soft tissue knee pathology. But where does meniscal allograft transplantation (MAT) fit in the treatment algorithm for patients with pain following loss of a functional meniscus? MAT has become an accepted method of treating post-meniscectomy pain, particularly in the young active individual with

Tim Spalding

tim@timspalding.com

Alan Getgood

alan.getgood@uwo.ca

1 University Hospitals Coventry and Warwickshire NHS Trust, Coventry, UK

2 Fowler Kennedy Sport Medicine Clinic, Western University, London, ON, Canada no signs of osteoarthritis [10]. For the younger patient with the worn osteoarthritic knee, in whom it is too early to opt for total or unicompartmental knee replacement, MAT is one of a number of surgical strategies utilized [3]. Terms such as biological arthroplasty and biological joint replacement have been coined to cover this growing field [2]. It is a popular concept with underlying hype for joint preservation, but strong clinical data and subsequent interpretation are critical to determine the real role for these complex techniques.

Unfortunately, the benefit of such treatments can be hard to quantify and there is lack of agreement as to how to define the appropriate expected outcome [4]. The importance of defining outcome is imperative when considering indications for MAT, balancing expectations against risk, in particular whether MAT can be considered protective for the articular surface, and as such a long-term joint preserving option. In light of the above of indications, we must consider what is the most appropriate method of reporting outcome of MAT. Is it patient function and quality of life? Is it prevention of OA? Or maybe implant/graft survival, similar to that of total knee arthroplasty?

MAT has been around for many years with 20-year results available [1]; however, as techniques and indications have evolved, the value of these data must be interpreted appropriately. MAT is now an arthroscopic-assisted procedure, passing the meniscus into the joint through extended portals and fixing it in place using a variety of soft tissue or bone fixation methods, with a combination of all inside and inside-out meniscal suture fixation techniques in the periphery. Compare this to the early techniques of the 1980s and 1990s, described in the long-term studies from Verdonk [14]. This involved an open surgical technique detaching the collaterals at the epicondyle, distracting the joint and laying in the fresh meniscal graft, suturing 
directly to the native meniscal remnants. Mechanically, this technique has been shown to be inferior to current fixation methods [7]. Indeed recent data from the results of transplants performed in Gent show improved survival in those treated with the arthroscopic technique (Verdonk personal communication). It is therefore clear that graft survivorship in long-term studies must be interpreted with caution, as techniques are often significantly different and therefore would be similar to comparing modern day TKA encompassing latest generation bearing surfaces and highly crosslinked polyethylene, to the early implant designs of the Freeman and Insall prostheses.

What about function and quality of life-does MAT improve patient reported outcome (PRO)? In a systematic review of PROs detailing 1332 patients and 1374 knees undergoing MAT, clear evidence of improvement in outcome was demonstrated, such that mean Lysholm scores were observed to improve to 81 , the IKDC subjective score improving to 70 and the Tegner activity scores improving to a mean of 4.7 , indicating a satisfactory level of work and recreational activity [11]. The mean reported failure rate was $10.6 \%$ at 4.8 years with outcome being shown to be related to the state of the joint surfaces. However, two studies showed greater than $50 \%$ of patients achieved prolonged good outcome even with advanced wear [3, 13].

Noyes [8] recently reported the long-term (minimum 10 years) outcome on 40 grafts in 38 patients. A modern generation bone fixation surgical technique was utilized with 15 patients having concomitant osteochondral grafts. The meniscal grafts were cryopreserved, but this technique has yet to be shown to be different to fresh frozen. Symptom improvement was observed with patients reporting improvements in the perception of the function of their knee, with $75 \%$ working and $72 \%$ undertaking light sport. Two endpoints detailing survivorship were used, the first of which included the standard endpoints of revision, graft removal, arthroplasty, osteotomy or pain on daily living. This gave survival as $88 \%$ at 5 years, $63 \%$ at 10 years and $40 \%$ at 15 years. A more severe endpoint was defined utilizing poor MRI signal data, meniscal extrusion and degeneration on X-ray down to bone on bone, on the basis that the role of the meniscus would be questionable with the presence of these findings. Survival was lower; but in 17 of those considered to be failures on these criteria, only four underwent revision/repeat surgery and this was at a mean time of 5 years. Thirteen were still coping and had not progressed to need arthroplasty. They concluded that MAT can be considered a good option for treating the post-meniscectomized knee, but is not curative.

The reason for this most likely lies within the unanswered question surrounding this subject area-is MAT chondroprotective? Biomechanical studies on contact pressures [7] and two pre-clinical animal models argue in favour. Furthermore, in a systematic review looking at radiological joint space narrowing after MAT, Smith et al. [12] observed $0.032 \mathrm{~mm}$ per year of narrowing, which is less than what would be expected in an OA control population. This represents weak evidence to support the hypothesis that MAT may reduce progression of OA, yet still does not support the use of MAT as a prophylactic procedure to prevent OA.

So what conclusions can we draw from this combined experience? Several systematic reviews have clearly shown a consistent improvement in PROs following MAT and that level of improvement is substantial. However, long-term data are suggestive of deterioration over time. Better outcome is clearly reported in younger patients and in those where there is less damage to the joint surfaces. Overall, meta-analyses tell us we can quote to our patients a figure of $80 \%$ survival at 10 years and somewhere between 20 and $40 \%$ survival at 20 years, but these data are based on a mixture of surgical techniques and principles of treatment.

It is therefore fair to assume that all meniscal transplants will eventually 'fail' in the future, particularly as the patients are young at the time of implantation. But is the concept of 'buying time' a valid one? Knee problems in the young often kick-start a progression of treatment, and as Roman Seil has so carefully outlined in his editorial in this issue, we should be aiming to help patients stay in the 'green zone'. When counselling patients regarding outcome, we therefore must address the issues of arthritis prevention versus improvements in quality of life, and this should be balanced with the inherent risks associated with the procedure.

The overall goal of MAT is to achieve symptom-free activities of daily living without swelling or pain. Light sport should be a bonus, with an overall ambition to conserve knee function. Patients should be warned of failure in the future and of the need for 'fine tuning' surgery in the short and midterm (up to $30 \%$ of cases) [6]. A 17-year-old presenting with pain and severe restriction in function and therefore quality of life is interested in the here and now, wanting to be active in sport and to be able to work. A fix that provides such outcome is arguably valid. But what if the graft fails at the age of 27 , having provided 10 good years of function? is this outcome declared as a failed procedure? To answer this question, it is imperative that future studies incorporate measures of quality of life, return to work and cost-effectiveness, as days off work and longterm non-operative measures have been shown to have a significant impact on direct and indirect healthcare costs [5]. A current randomized trial of MAT against personalized knee therapy will hopefully provide data to answer this important question [9].

There is agreement that the successful treatment of the symptomatic post-meniscectomy knee in younger athletic 
patients is one of the highest priorities in orthopaedics that requires scientific advancement. A knee without a functional congruent meniscus is a compromised organ-biomechanically and biologically. It will ultimately deteriorate at both the articular cartilage and subchondral bone levels. Our responsibility as surgeons and researchers is to make every effort to restore meniscal function in the least invasive, yet optimal biomechanical and biologically sound manner. This 'mechanobiological' approach, along with addressing malalignment, ligament instability, subchondral bone and articular cartilage lesions, continues to evolve and challenges us.

Importantly, we should not burn bridges in our treatment pathway. Unlike the definitive 'bridge burning' nature of joint arthroplasty, MAT is a bridging procedure. Maybe the bridge will last for 10-15 years, but the key outcome is optimized function and comfort for as long as possible. In teenagers and those under 40 , there are few other good options-if we can delay their first arthroplasty by 20 years, we may save them from a revision TKA in their lifetime.

Time gained should therefore not be considered a failure, but should be considered as achieving pre-defined expectations, especially in a young population where there are no other options. Whilst research continues to attempt to answer the challenge to help the patient with post-meniscectomy syndrome, the glass should be considered half full ready for replenishment with new knowledge.

\section{References}

1. Elattar M, Dhollander A, Verdonk R, Almqvist KF, Verdonk $P$ (2011) Twenty-six years of meniscal allograft transplantation: is it still experimental? A meta-analysis of 44 trials. Knee Surg Sports Traumatol Arthrosc 19(2):147-157. doi:10.1007/ s00167-010-1351-6

2. Harris JD, Hussey K, Wilson H, Pilz K, Gupta AK, Gomoll A, Cole BJ (2014) Biological knee reconstruction for combined malalignment, meniscal deficiency, and articular cartilage disease. Arthroscopy. doi:10.1016/j.arthro.2014.08.012

3. Kempshall PJ, Parkinson B, Thomas M, Robb C, Standell H, Getgood A, Spalding T (2014) Outcome of meniscal allograft transplantation related to articular cartilage status: advanced chondral damage should not be a contraindication. Knee Surg Sports Traumatol Arthrosc. doi:10.1007/s00167-014-3431-5

4. LaPrade CM, James EW, LaPrade RF, Engebretsen L (2015) How should we evaluate outcomes for use of biologics in the knee? J Knee Surg 28(1):35-44. doi:10.1055/s-0034-1390028

5. Mather RC 3rd, Hettrich CM, Dunn WR, Cole BJ, Bach BR Jr, Huston LJ, Reinke EK, Spindler KP (2014) Cost-effectiveness analysis of early reconstruction versus rehabilitation and delayed reconstruction for anterior cruciate ligament tears. Am J Sports Med 42(7):1583-1591. doi:10.1177/0363546514530866

6. McCormick F, Harris JD, Abrams GD, Hussey KE, Wilson H, Frank R, Gupta AK, Bach BR Jr, Cole BJ (2014) Survival and reoperation rates after meniscal allograft transplantation: analysis of failures for 172 consecutive transplants at a minimum 2-year follow-up. Am J Sports Med 42(4):892-897. doi:10.1177/0363546513520115

7. McDermott ID, Lie DT, Edwards A, Bull AM, Amis AA (2008) The effects of lateral meniscal allograft transplantation techniques on tibio-femoral contact pressures. Knee Surg Sports Traumatol Arthrosc 16(6):553-560. doi:10.1007/ s00167-008-0503-4

8. Noyes FR, Barber-Westin SD (2015) Meniscal transplantation in symptomatic patients under fifty years of age: survivorship analysis. J Bone Joint Surg Am 97(15):1209-1219. doi:10.2106/ JBJS.N.01340

9. Smith NA, Achten J, Parsons N, Wright D, Parkinson B, Thompson P, Hutchinson CE, Spalding T, Costa ML (2015) Meniscal transplantation and its effect on osteoarthritis risk: an abridged protocol for the MeTEOR study: a comprehensive cohort study incorporating a pilot randomised controlled trial. Bone Joint Res 4(6):93-98. doi:10.1302/2046-3758.46.2000318

10. Smith NA, Costa ML, Spalding T (2015) Meniscal allograft transplantation: rationale for treatment. Bone Joint $\mathrm{J}$ 97-B(5):590-594. doi:10.1302/0301-620X.97B5.35152

11. Smith NA, MacKay N, Costa M, Spalding T (2015) Meniscal allograft transplantation in a symptomatic meniscal deficient knee: a systematic review. Knee Surg Sports Traumatol Arthrosc 23(1):270-279. doi:10.1007/s00167-014-3310-0

12. Smith NA, Parkinson B, Hutchinson CE, Costa ML, Spalding T (2015) Is meniscal allograft transplantation chondroprotective? A systematic review of radiological outcomes. Knee Surg Sports Traumatol Arthrosc. doi:10.1007/s00167-015-3573-0

13. Stone KR, Pelsis JR, Surrette ST, Walgenbach AW, Turek TJ (2015) Meniscus transplantation in an active population with moderate to severe cartilage damage. Knee Surg Sports Traumatol Arthrosc 23(1):251-257. doi:10.1007/s00167-014-3246-4

14. Verdonk R, Van Daele P, Claus B, Vandenabeele K, Desmet P, Verbruggen G, Veys EM, Claessens H (1994) Viable meniscus transplantation. Orthopade 23(2):153-159 